\title{
Performance evaluation of an acoustic indoor localization system based on a fingerprinting technique
}

\author{
Nadia Aloui ${ }^{1,2^{*}}$, Kosai Raoof ${ }^{3}$, Ammar Bouallegue $^{2}$, Stephane Letourneur ${ }^{3}$ and Sonia Zaibi ${ }^{2}$
}

\begin{abstract}
We present an acoustic location system that adopts the time of arrival of the path of maximum amplitude as a signature and estimates the target position through nonparametric kernel regression. The system was evaluated in experiments for two main configurations: a privacy-oriented configuration with code division multiple access operation and a centralized configuration with time division multiple access operation. The effects of the number and positions of sources on the performance of the privacy-oriented system was studied. Moreover, the effect of the number of fingerprint positions on the performance of both systems was investigated. Results showed that our privacy-oriented scheme provides an accuracy of $8.5 \mathrm{~cm}$ with $87 \%$ precision, whereas our centralized system provides an accuracy of $2.7 \mathrm{~cm}$ for $93 \%$ of measurements. A comparison between our privacy-oriented system and another acoustic location system based on code division multiple access operation and lateration was conducted on our test bench and revealed that the cumulative error distribution function of the fingerprint-based system is better than that of the lateration-based system. This result is similar to that found for Wi-Fi radio-based localization. However, our experiments are the first to demonstrate the detrimental effect that reverberation has on naive acoustic localization approaches.
\end{abstract}

Keywords: Acoustic; Fingerprinting technique; Nonparametric kernel regression; Code division multiple access operation; Time division multiple access operation; Privacy-oriented configuration; Centralized system; Lateration

\section{Introduction}

Indoor location estimation has various useful applications in everyday life. For instance, in hospitals, doctors can rapidly reach their patients using their location information. In a conference, a journalist can be localized by an indoor positioning system so that he can be guided to find the right conference room. Property and equipment can also be protected by indoor location systems through monitoring and tracking.

Several indoor location systems have been developed in the last decade. They are mainly based on triangulation

\footnotetext{
*Correspondence: nadia.aloui@gipsa-lab.grenoble-inp.fr

1 Grenoble Images Parole Signal Automatique (GIPSA-lab) CNRS : UMR5216 Université Joseph Fourier - Grenoble I - Université Pierre-Mendès-France Grenoble II - Université Stendhal - Grenoble III - Institut Polytechnique de Grenoble (Grenoble Institute of Technology), Grenoble, France

2 Université de Tunis El Manar, Ecole Nationale d'Ingénieurs de Tunis,

LR-99-ES21 Laboratoire de Systèmes de Communications, Tunis 1002, Tunisie

Full list of author information is available at the end of the article
}

and fingerprinting techniques [1]. Triangulation has two derivatives: angulation and lateration. Both require the line of sight between the transmitter and receiver. Lateration needs at least three measured distances between the target and references of known positions to calculate the two-dimensional (2D) location of the target. The angulation estimates a target's $2 \mathrm{D}$ position from the measured angles between the target and at least two known locations. On the other hand, the fingerprinting technique frames the positioning problem as a supervised learning problem. First, a set of positions, called fingerprint positions (or sampling locations), is selected in the target environment, and then, a position-dependent signal parameter is extracted at each position to define the position signature (also called the fingerprint). A fingerprint database is then constructed. Second, the target signature is calculated then compared with signatures in the previously built database to estimate the position.

\section{Springer}

(c) 2014 Aloui et al.: licensee Springer. This is an Open Access article distributed under the terms of the Creative Commons

Attribution License (http://creativecommons.org/licenses/by/2.0), which permits unrestricted use, distribution, and reproduction in any medium, provided the original work is properly cited. 
In this work, we present a novel indoor localization system that is based on the fingerprinting technique. The system provides location estimation within a room. It can be applied to Alzheimer's patients who may forget where they have put their property. Equipped with a sound receiver or emitter, patient positions are estimated and recorded in real time. Therefore, patients may review their displacements in the house.

The system performance is assessed in two different configurations: privacy-oriented configuration with code division multiple access (CDMA) operation and a centralized configuration with time division multiple access (TDMA) operation. For the privacy-oriented system, we propose to select the most robust elements in a signature to improve the performance. We believe that this is the first time that the approach has been proposed.

The following section gives a brief overview of indoor location systems. The proposed system is described in Section 3. Section 4 presents the materials and parameters of experiments. The experimental results obtained for the privacy-oriented configuration and the centralized configuration are analyzed in Sections 5 and 6. Limitations and future work are discussed in Section 7. The last section concludes the paper.

\section{Indoor location systems}

The most popular location system is the global positioning system (GPS), which has good performance outdoors but fails indoors. To overcome the GPS limits, other systems have been developed for indoor localization. These systems can be classified according to several aspects.

Conceptually, they can be categorized into centralized systems and privacy-oriented systems. In the centralized systems [2], a central service gathers the location information of the target and employs it to estimate the position. In such a system, target privacy can be violated since the location information is recorded without explicit permission. Privacy-oriented systems preserve user privacy. Therefore, they must have two properties [2]. The first is that the target does not need to inform external devices about its presence. The second is that the devices are not responsible for collecting location data or calculating the target position. Therefore, the target estimates its position by itself.

Location systems can be polled, meaning that the transmitters are told when to emit their messages [2]. The Dolphin system [2] is an example of a polled, centralized system and a privacy-oriented system. Similarly to the GPS, the Dolphin system deploys the direct sequence CDMA method to obtain simultaneous distance measurements and employs multilateration to estimate position. The system deploys ultrasound signals and has accuracy of $2.2 \mathrm{~cm}$ at the $95 \%$ confidence level for $93 \%$ of valid readings in the polled and centralized mode and has accuracy of $4.9 \mathrm{~cm}$ for $67 \%$ of returned readings in the synchronous privacy-oriented mode.

According to the type of emitted signal, indoor location systems can be classified into infrared (IR) positioning systems, radiofrequency (RF) systems, and acoustic systems.

\subsection{IR systems}

IR positioning systems require line of sight between transmitters and receivers and provide precise positioning estimations. They have a simple architecture and therefore do not need much time for installation and maintenance. Nevertheless, they suffer from the sensitivity of IR signals to interference from fluorescent light and sunlight [3]. This problem can be resolved using optical and electronic filters [4] and implementing a noise canceling algorithm at the receivers [3]. However, this raises the system cost.

An active badge system is an example of an IR-based location system. The system comprises an active badge, sensors, and a central server. The active badge attached to a person emits a unique IR signal every $15 \mathrm{~s}$ [5]. Once the emitted signal is detected, the location information is forwarded to the central server to locate the active badge.

\subsection{RF systems}

RF positioning systems have a large coverage area because radio waves can pass through walls. The systems mainly use triangulation and fingerprinting techniques for localization and have the advantage of employing existing $\mathrm{RF}$ infrastructure. In [6], the complex channel impulse response (CIR) was collected using a channel sounder. The time impulse response is obtained by applying the inverse fast Fourier transform (IFFT). Seven parameters are then extracted from each CIR to form a signature, and an artificial neural network is trained to estimate the target position in underground areas. The system provides good localization accuracy. However, the deployed devices are costly and heavy. In [7], the system is designed for users of wideband orthogonal frequency division multiplexing technologies. The CIR is approximated by performing an IFFT on the receiver's channel estimation. The amplitudes of the approximate CIR vector (ACIR) define the signature, and the target position is estimated through nonparametric kernel regression. The system offers better localization accuracy than other fingerprintbased methods, such as methods employing the received signal strength fingerprint with kernel distance [8] and the decimal-scale ACIR fingerprint with general regression neural networks generalized from [9]. However, the fingerprint dimension per access point is fixed to 128 elements, which may be time-consuming.

\subsection{Acoustic systems}

In the cricket system $[10,11]$, the times of arrival (TOAs) of ultrasound signals from the emitters at known positions 
to the receiver to be located are measured. The position is estimated through triangulation. The RF signals are employed for synchronization of TOA measurements. In [12], the three-dimensional (3D) locus system is described. The system employs ultrasound signals that are based on binary phase shift keying (BPSK)-modulated spreading Golay codes. Three configurations have been considered in evaluating system performance: centralized, privacy-oriented, and bidirectional configurations. Four test conditions have been evaluated for each configuration: TDMA with calm air, CDMA with calm air, TDMA with airflow, and TDMA with airflow. The system provides subcentimeter accuracy.

The ambient sound has been exploited for localization in [13] and [14]. The authors (SurroundSense) of [13] have combined sound, light, color, and acceleration that can be sensed by a microphone, camera, and accelerometer of a phone to construct an identifiable fingerprint. Existing Wi-Fi-based fingerprinting is also included as the fifth subfingerprint. SurroundSense devices obtained from 51 different stores had an average accuracy of $87 \%$.

The system proposed in [14] uses an ambient sound fingerprint called the acoustic background spectrum (ABS). Unlike SurroundSense's acoustic fingerprint, which is a compact histogram of a sound recording from the time domain, the ABS system employs a compact spectral representation [14]. The system aims to locate a basic mobile device with room resolution employing nearest-neighbor classification. Combined with a Wi-Fi-based localization method, ABS localization improves the accuracy from $30 \%$ (Wi-Fi only) to $69 \%$ (Wi-Fi and ABS). A comparison with SurroundSense [13] reveals that the ABS [14] system has higher performance when only the microphone is used.

Our earlier proposed system, presented in [15], is similar to the Dolphin system [2], except that it adopts trilateration instead of multilateration and audible sound instead of an ultrasound signal. The system deploys four tweeters and aims to locate a microphone. Two methods have been proposed. The first method uses four combinations of three of the four measured distances to generate four estimates of the microphone location. The resulting position is the center point of the four estimates. The second method uses the three most reliable distances for the computation of the microphone position. In [15], the first method was adopted and the system gives accuracy of 2 $\mathrm{cm}$ with $99 \%$ precision. Inspired from [15], a novel acoustic localization system was presented in [16]. The system is a receiver localization system that uses CDMA operation. It differs from the system described in [15] in that it uses the fingerprinting technique instead of lateration. It deploys three tweeters and computes the microphone position through nonparametric kernel regression.

In this paper, we will enrich the location system presented in [16]. We will investigate the effect of the fingerprint positions on system performance. We will then evaluate the cumulative error distribution of the system while varying the number and positions of speakers. Third, we will assess the system performance in a centralized configuration and with TDMA operation.

\section{Proposed system}

We propose a fingerprint-based localization system that adopts the TOAs of signals from transmitter(s) to receiver(s) as a fingerprint and computes the target position through the local linear estimator. The system has two configurations: a privacy-oriented configuration and centralized configuration. The first aims to locate a microphone using CDMA operation, whereas the second aims to estimate the speaker's position using TDMA operation.

\subsection{CDMA method}

In a direct sequence spread spectrum system, a data signal is multiplied by a pseudorandom binary sequence. The

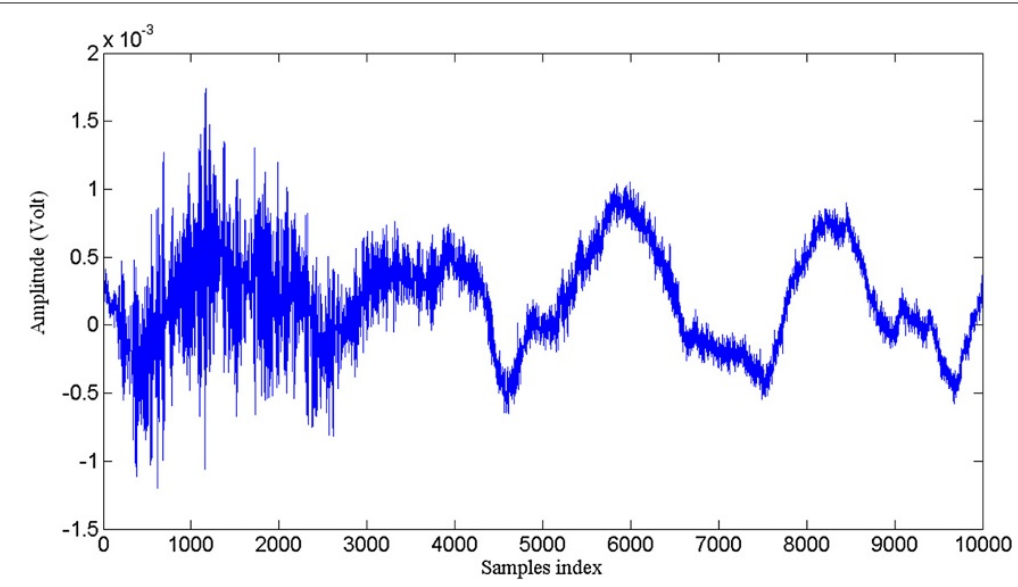

Figure 1 Received signal in the case of a privacy-oriented system. 


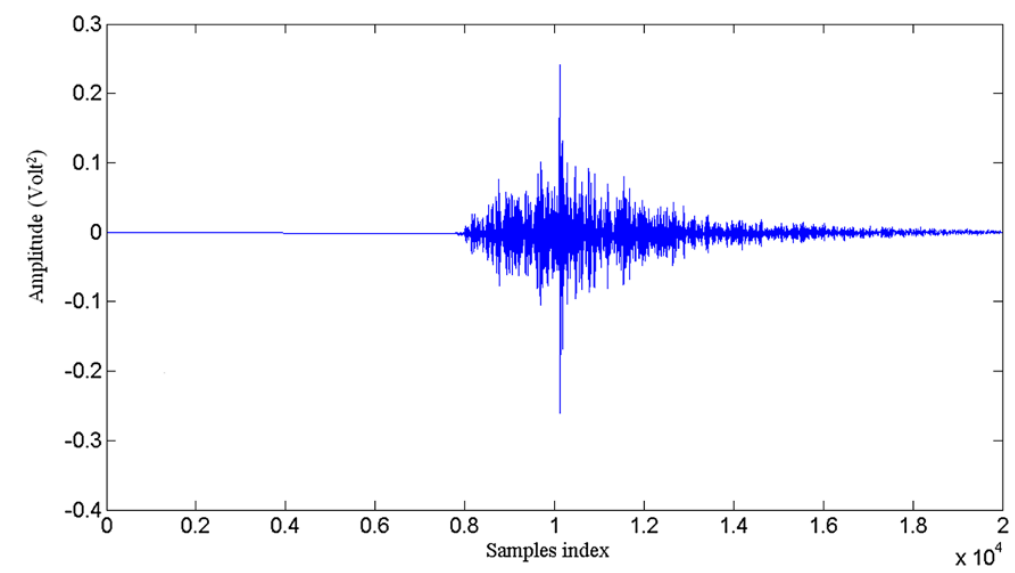

Figure 2 Correlation between the received and transmitted signals in the case of a privacy-oriented system.

bandwidth of the modulated signal is larger than that of the original data signal. One advantage of this spreading spectrum is that the resulting signal is more resistant to narrow-band interference.

Different spread spectrum sequences have been developed, such as m-sequences, orthogonal codes, and gold codes. The m-sequences have good autocorrelation and cross-correlation properties. However, they are generated by primitive polynomials and thus generated in small number. Orthogonal codes do not have good autocorrelation properties and their cross-correlation is equal to zero only when there is no lag between codes. Therefore, they are adopted for perfectly synchronized systems. Gold codes are recognized for their good autocorrelation and cross-correlation properties. Moreover, they are generated in large number. The desirable cross-correlation properties of gold codes allow multiple devices to transmit simultaneously in the same frequency range, while their desirable autocorrelation properties enable code synchronization. Therefore, these codes allow accurate and simultaneous time measurements.

Nevertheless, CDMA systems suffer from the near-far effect, which corresponds to the difference in received power of the users' signals. In fact, the autocorrelation peak of the weaker signal can be masked by the significant cross-correlation peaks owing to the other stronger signals, and it is thus difficult for the receiver to detect the weaker signal.

In this paper, gold codes are adopted and no power control is implemented to avoid the near-far effect. In the following section, we present the fingerprint that we have adopted in our system.

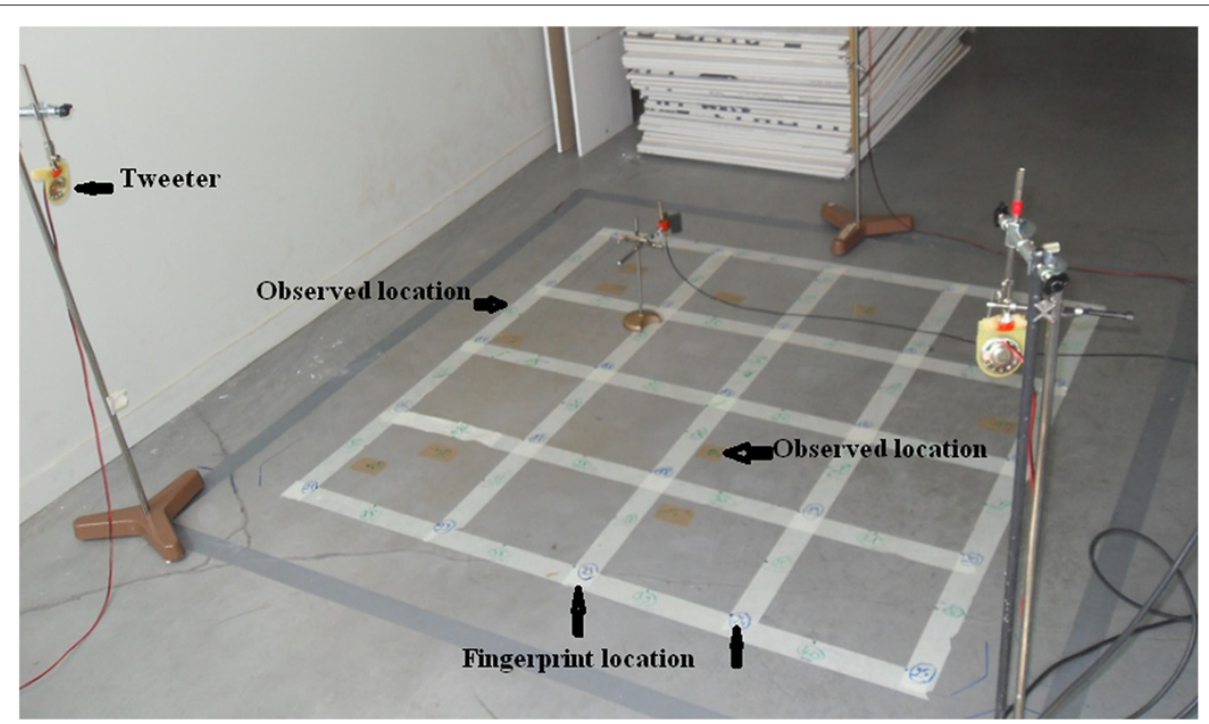

Figure 3 Test platform for the privacy-oriented configuration. 


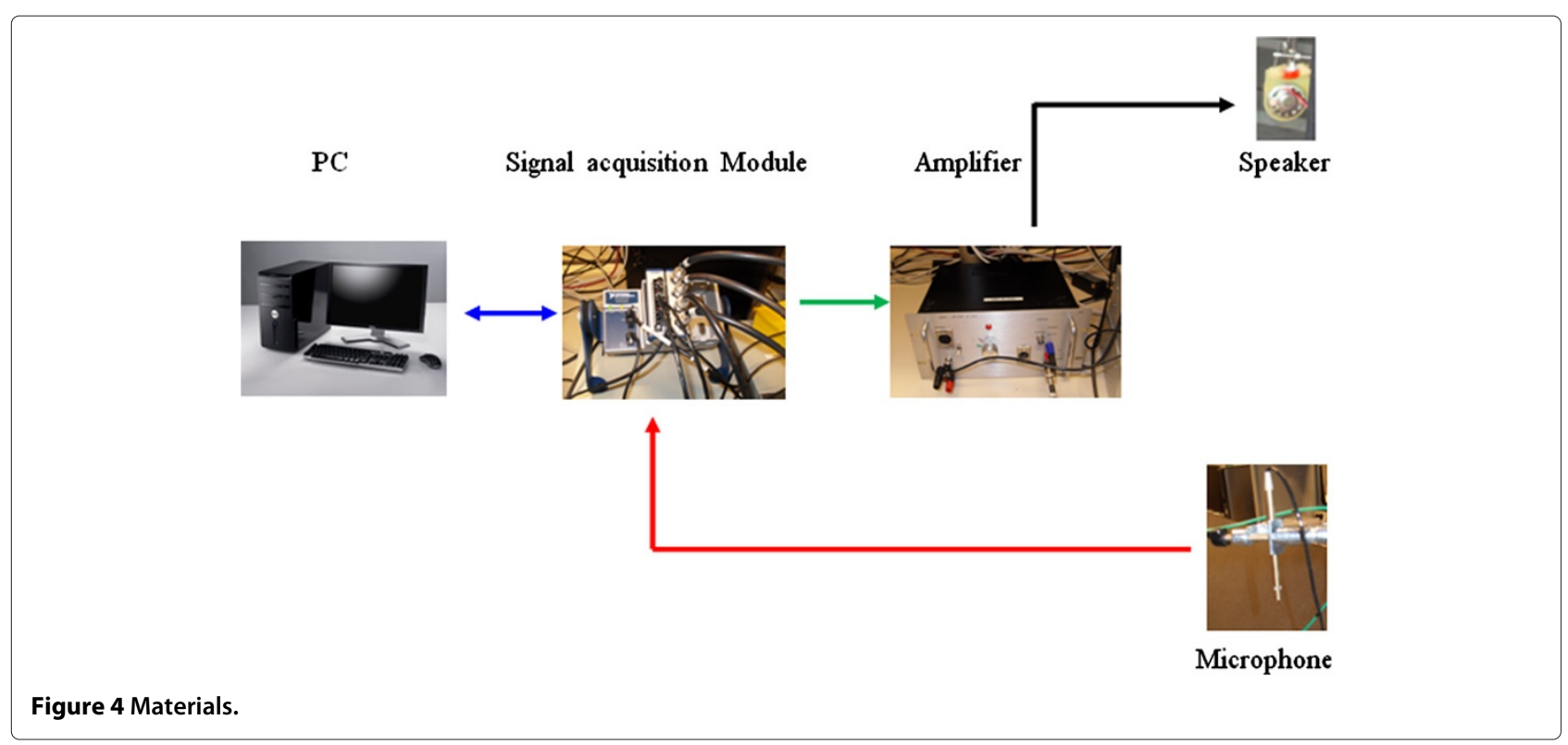

\subsection{TOA-based fingerprint}

The TOA of a signal of maximum amplitude depends on the location of the emitter and receiver. For a receiver (emitter) of known position, the TOA characterizes the unknown position of the emitter (receiver). In this paper, we adopt the TOA as a fingerprint.

To estimate the TOA, the receiver, which is synchronized with emitters, correlates the received signal
(Figure 1) with the emitted codes. The instant of the peak correlation value obtained for each code corresponds to an estimate of the TOA (see Figure 2). The obtained TOAs represent the target signature. The signature dimension, $d$, is then equal to the number of deployed sources in the privacy-oriented configuration. For the centralized configuration, $d$ is equal to the number of receivers.
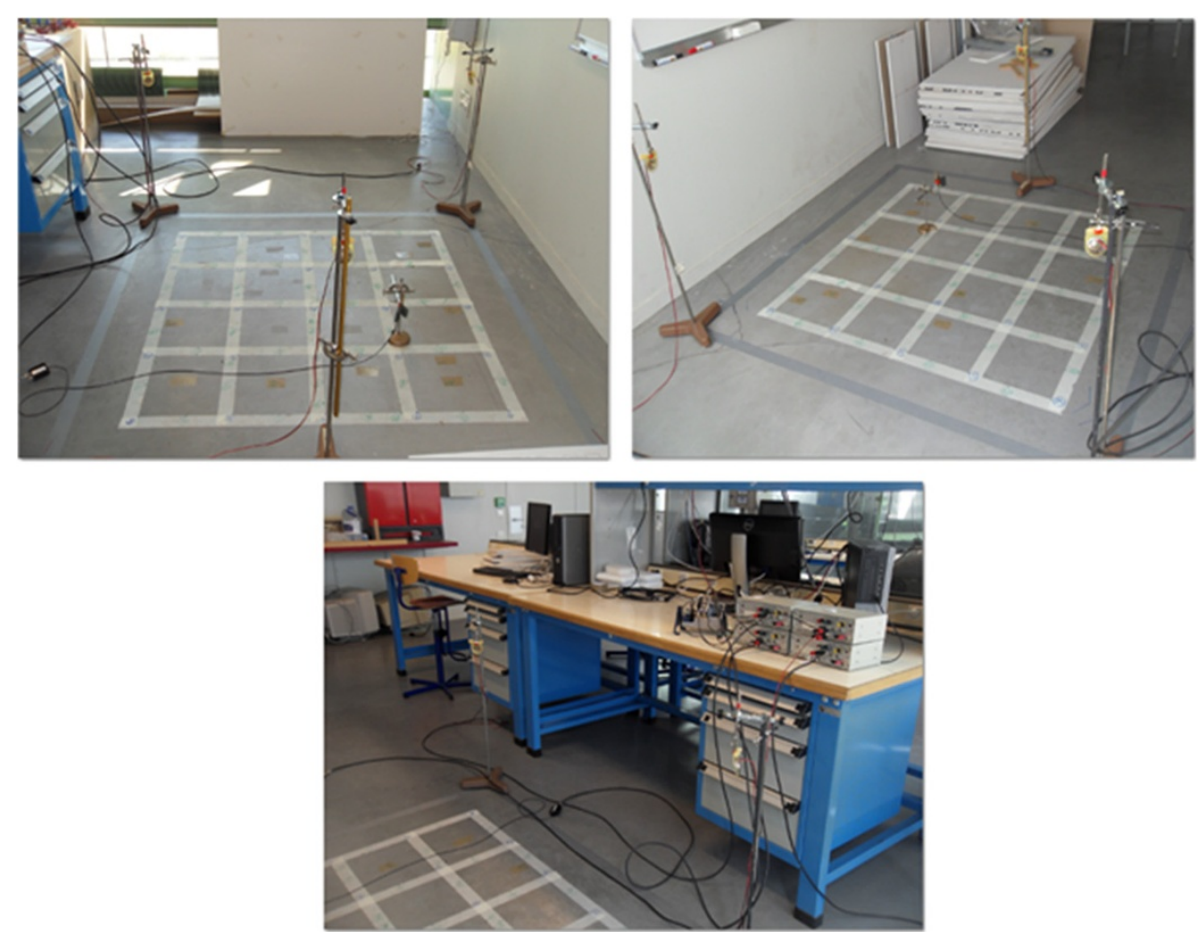

Figure 5 Measurement environment. 


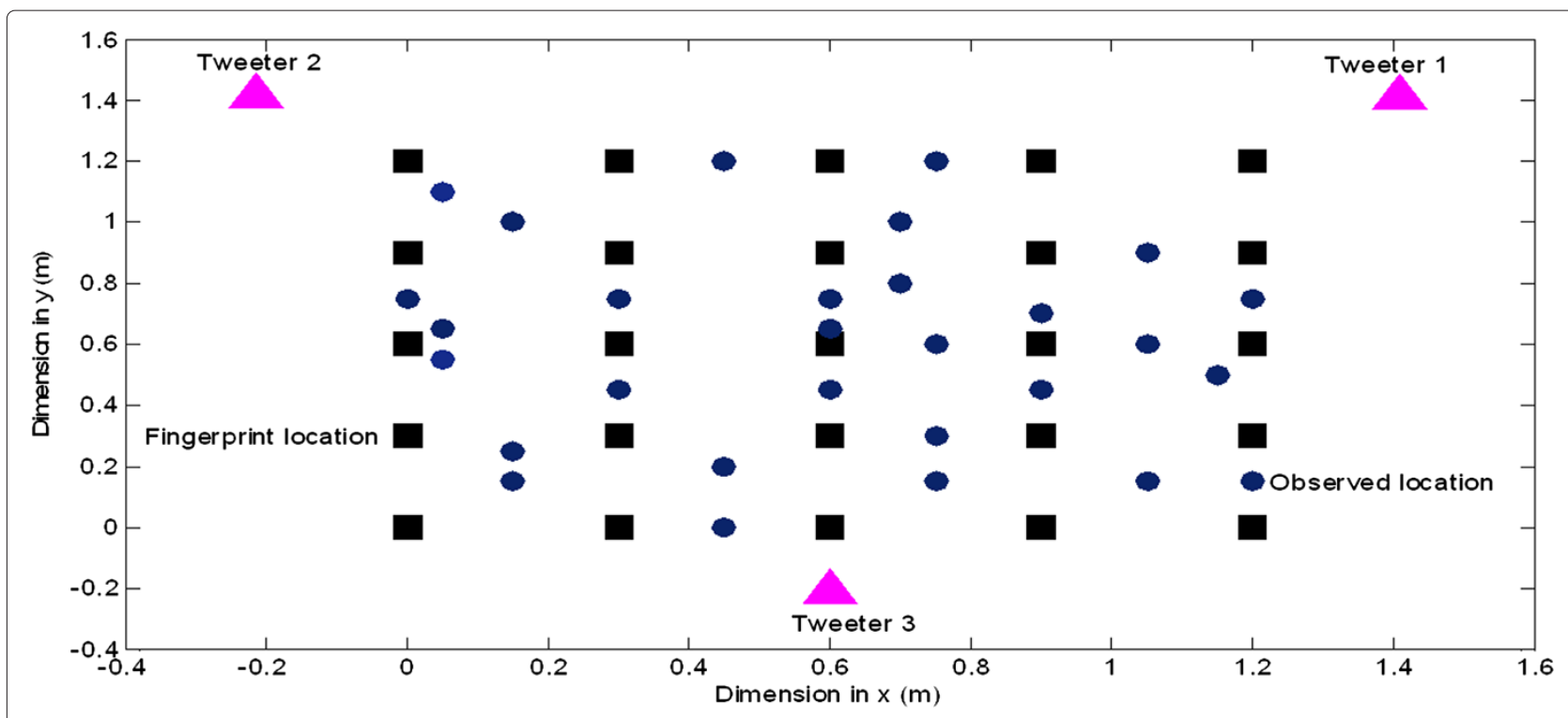

Figure 6 Layout of the first experiment setup in the case of the first configuration.

The process of TOA estimation is repeated for the L fingerprint positions selected in the target environment and shown in Figure 3 . The $L$ resulting signatures $\left(s_{l}\right)_{1<=l<=L}$ and the corresponding positions $\left(c_{l}\right)_{1<=l<=L}$ are stored in a database.

In the online phase, the target signature is compared with the stored signatures through a nonparametric kernel estimator.

\subsection{Local linear estimator}

We aim to estimate the 2D position of the target $c$ whose fingerprint is $s$. This is equivalent to estimating the con- ditional expectation $E(c \mid s)$, denoted $m(s)$ and defined as:

$$
E(c \mid s)=\frac{\int c \times f(c, s) d c}{\int f(c, s) d c}
$$

where $f(c, s)$ denotes the joint probability density function. Probabilistic localization methods, such as that presented in [7], use the kernel approach to approximate the conditional expectation $E(c \mid s)$.

In the following, we will explain the principle of local polynomial kernel fits in the case of univariate variables

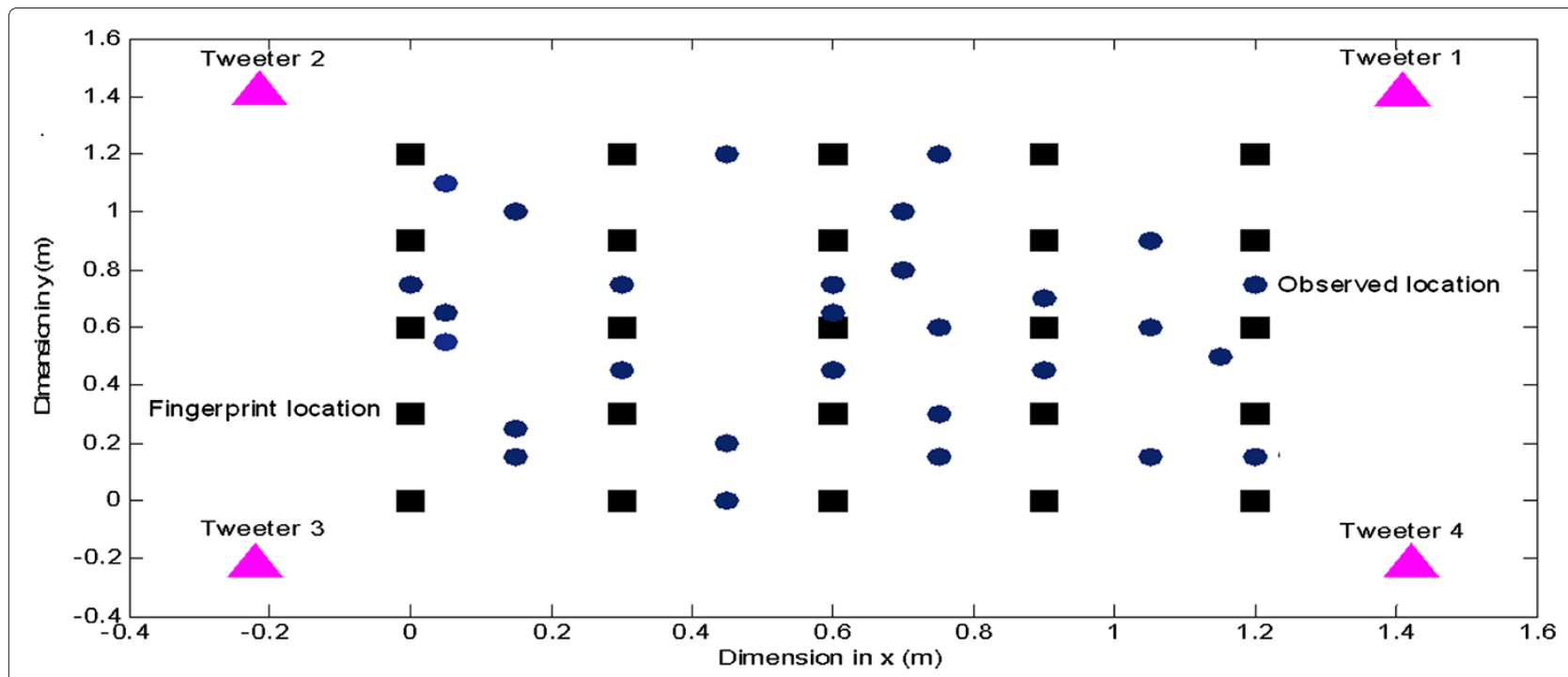

Figure 7 Layout of the second experiment setup in the case of the first configuration. 
Table 1 Positioning accuracy and precision obtained with the proposed scheme in the case of the first configuration

\begin{tabular}{lccccc}
\hline \multicolumn{5}{c}{ Experimental results } \\
\hline Error distance (cm) & 5 & 6 & 8.5 & 12 & 15 \\
Precision (\%) & 55.66 & 66.5 & 80.29 & 90.64 & 94.08 \\
\hline
\end{tabular}

(i.e., $d=1$ ). We will then consider the case of a local linear estimator for $d>1$.

\subsubsection{Univariate case}

To motivate the local polynomial fits, let us consider the Taylor expansion of order $p$ of the unknown conditional expectation function $m($.$) :$

$m(t)=m(x)+m^{(1)}(x) \times(t-x)+\ldots+m^{(p)}(x) \times(t-x)^{p} \times \frac{1}{p !}$

for $t$ in a neighborhood of a point $x . m^{(i)}(x)$ denotes the $i$ th order derivative of the function $m($.).

This corresponds to a polynomial fit in a neighborhood of $x$. Kernel weights are included in the minimization problem to realize the neighborhood:

$$
\begin{aligned}
\min _{\beta} & \sum_{i=1}^{L}\left(c_{i}-\beta_{0}-\beta_{1} \times\left(s_{i}-s\right)-\ldots\right. \\
& \left.-\beta_{p} \times\left(s_{i}-s\right)^{p}\right)^{2} \times K_{h}\left(s_{i}-s\right),
\end{aligned}
$$

where $\beta=\left(\beta_{0}, \beta_{1}, \ldots, \beta_{p}\right)^{T}$ is the coefficient vector, $s_{i}$ is the signature of the fingerprint location $i$, and $K_{h}\left(s_{i}-s\right)$ is the kernel weight defined as:

$$
K_{h}(.)=\frac{1}{h} \times K\left(h^{-1}(.)\right) .
$$

Here, $K$ is a kernel function and $h$ is the bandwidth.
The solution of the minimization problem (3) is a weighted least squares estimator with weights $K_{h}\left(s_{i}-s\right)$ given by:

$$
\tilde{\beta}(s)=\left(S^{T} W S\right)^{-1}\left(S^{T} W C\right),
$$

where

$$
\begin{aligned}
C & =\left(\begin{array}{c}
c_{1} \\
c_{2} \\
\vdots \\
c_{L}
\end{array}\right) \\
S & =\left(\begin{array}{c}
1\left(s_{1}-s\right)\left(s_{1}-s\right)^{2} \ldots\left(s_{1}-s\right)^{p} \\
1\left(s_{2}-s\right)\left(s_{2}-s\right)^{2} \ldots\left(s_{2}-s\right)^{p} \\
\ldots \ldots \\
1\left(s_{L}-s\right)\left(s_{L}-s\right)^{2} \ldots\left(s_{L}-s\right)^{p}
\end{array}\right)
\end{aligned}
$$

and

$$
W=\operatorname{diag}\left(K_{h}\left(s_{1}-s\right), \ldots, K_{h}\left(s_{L}-s\right)\right) .
$$

Comparing Equations (2) and (3), the local linear polynomial estimator of the regression function $m($.) is determined to be:

$$
\tilde{m}(s)=\tilde{\beta}_{0}(s) .
$$

The orders $p=1$ and $p=3$, that correspond to the local linear estimator and local cubic regression, are usually used in estimating regression functions [17]. In [17], it was demonstrated that odd order fits outperform even order fits.

\subsubsection{Multivariate case}

The multivariate local polynomial kernel regression is a straightforward generalization of the univariate case. Let

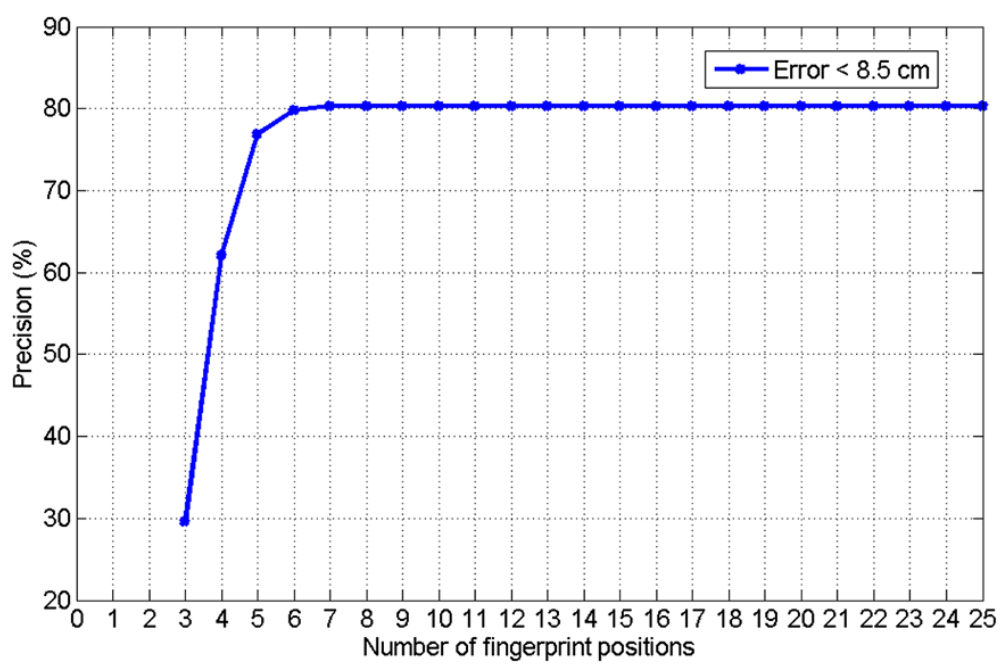

Figure 8 Precision for $8.5-\mathrm{cm}$ accuracy versus number of fingerprint positions obtained in the first configuration. 


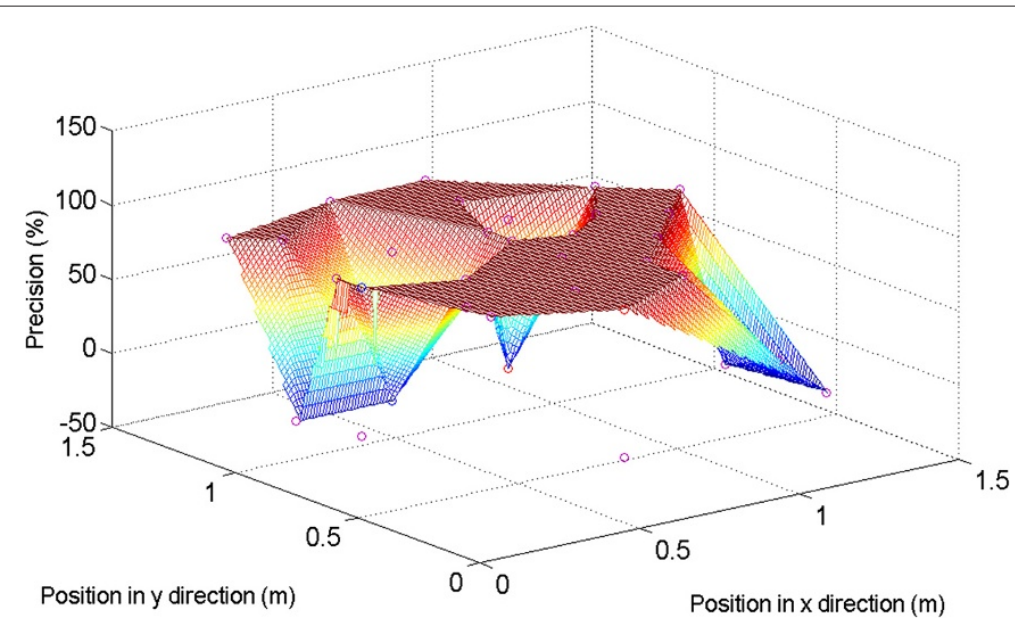

Figure 9 Precision distribution for $8.5-\mathrm{cm}$ accuracy related to the first configuration using $M=7$.

us consider the case of a local linear regression estimate; i.e., $p=1$. The minimization problem is then given as:

$$
\min _{\beta_{0}, \beta_{1}} \sum_{i=1}^{L}\left(c_{i}-\beta_{0}-\beta_{1}^{T} \times\left(s_{i}-s\right)\right)^{2} \times K_{H}\left(s_{i}-s\right) .
$$

The solution to this problem can be written as:

$$
\tilde{\beta}(s)=\left(S^{T} W S\right)^{-1}\left(S^{T} W C\right),
$$

where

$$
C=\left(\begin{array}{c}
c_{1} \\
\vdots \\
c_{L}
\end{array}\right)
$$

$$
S=\left(\begin{array}{c}
1\left(s_{1}-s\right)^{T} \\
\vdots \vdots \\
1\left(s_{L}-s\right)^{T}
\end{array}\right)
$$

and

$$
W=\operatorname{diag}\left(K_{H}\left(s_{1}-s\right), \ldots, K_{H}\left(s_{L}-s\right)\right) .
$$

$K_{H}$ is the kernel weight defined as:

$$
K_{H}(.)=\frac{1}{\operatorname{det}(H)} \times K\left(H^{-1}(.)\right) .
$$

$K$ is the multivariate kernel function, and $H$ is the bandwidth matrix. The kernel function is usually a probability density function [17]. Its value, $K(u)$, increases as $u$ approaches zero. The bandwidth matrix $H$ controls the orientation and the shape of the kernel function.

In [7], the authors adopted the Nadaraya-Watson estimator (i.e., an estimator with order $p=0$ ) to estimate

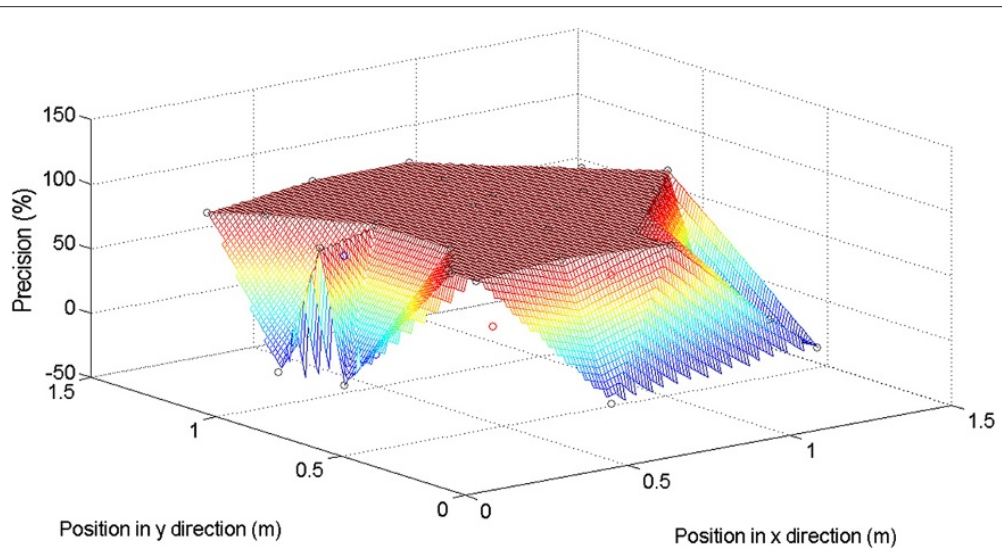

Figure 10 Precision distribution for $8.5-\mathrm{cm}$ accuracy related to the first configuration using $M=10$. 
the positions of users of both existing and upcoming wideband OFDM technologies.

In this paper, we employ the local linear estimator (i.e., $p=1$ ). We have selected the minimum odd order to reduce the time computation of the target position. We have also adopted the Gaussian kernel function defined as:

$$
K(u)=\frac{1}{(2 \times \Pi)^{\frac{d}{2}}} \times \exp \left[-\frac{1}{2} \times u^{T} \times u\right]
$$

and the bandwidth $H$ derived from the multivariate generalization of Scott's rule of thumb for bandwidth selection as [17]

$$
H=L^{\frac{-1}{(d+4)}} \times \mathrm{Co}^{\frac{1}{2}},
$$

where Co is the sample covariance matrix of the fingerprint vectors $s_{1}, s_{2}, \ldots, s_{L}$ and $d$ is the dimension of the fingerprint vector.

Using the notations

$$
\begin{aligned}
& S_{0}=\sum_{i=1}^{L} K_{H}\left(s_{i}-s\right), \\
& S_{1}=\sum_{i=1}^{L} K_{H}\left(s_{i}-s\right) \times\left(s_{i}-s\right), \\
& S_{2}=\sum_{i=1}^{L} K_{H}\left(s_{i}-s\right) \times\left(s_{i}-s\right) \times\left(s_{i}-s\right)^{T}, \\
& T_{0}=\sum_{i=1}^{L} K_{H}\left(s_{i}-s\right) \times c_{i}, \\
& T_{1}=\sum_{i=1}^{L} K_{H}\left(s_{i}-s\right) \times\left(s_{i}-s\right) \times c_{i},
\end{aligned}
$$

the local linear estimator of the position $c$ can be equivalently written as [18]:

$$
\tilde{c}=\frac{T_{0}-S_{1}^{T}-S_{2}^{-1} T_{1}}{S_{0}-S_{1}^{T}-S_{2}^{-1} S_{1}} .
$$

Note that $S 1$ and $T 1$ are $d$-variate vectors and $S 2$ is a $d \times d$ matrix.

In the following, we describe the materials that we have employed and the test bench on which we have made measurements.

\section{Materials and experiment environment}

The hardware system is composed of a signal acquisition module, an amplifier, four speakers, four microphones, and a personal computer (PC) (see Figure 4). The signal acquisition module deploys $q$ uniform output channels to broadcast the $q$ signals generated by the $\mathrm{PC}$, and $u$ uniform input channels to record the $u$ received signals. Depending on the system configuration and setup, $q$ will take the values 1,3 , and 4 , and $u$, the values 1 and 4 . The sensitivity of the deployed microphones is about 10 $\mathrm{mV} / \mathrm{Pa}$.

Data acquisition between the $\mathrm{PC}$ and the signal acquisi-

\begin{tabular}{|c|c|c|}
\hline Positions & $\begin{array}{c}\text { Precision (\%) obtained } \\
\text { with } M=7\end{array}$ & $\begin{array}{c}\text { Precision (\%) obtained } \\
\text { with } M=10\end{array}$ \\
\hline Position 1 & 100 & 0 \\
\hline Position 2 & 0 & 0 \\
\hline Position 3 & 100 & 100 \\
\hline Position 4 & 100 & 100 \\
\hline Position 5 & 100 & 100 \\
\hline Position 6 & 100 & 100 \\
\hline Position 7 & 100 & 100 \\
\hline Position 8 & 100 & 100 \\
\hline Position 9 & 0 & 0 \\
\hline Position 10 & 0 & 100 \\
\hline Position 11 & 100 & 100 \\
\hline Position 12 & 100 & 100 \\
\hline Position 13 & 100 & 100 \\
\hline Position 14 & 100 & 100 \\
\hline Position 15 & 100 & 100 \\
\hline Position 16 & 100 & 100 \\
\hline Position 17 & 100 & 100 \\
\hline Position 18 & 100 & 100 \\
\hline Position 19 & 100 & 100 \\
\hline Position 20 & 28.57 & 28.57 \\
\hline Position 21 & 100 & 0 \\
\hline Position 22 & 0 & 0 \\
\hline Position 23 & 100 & 100 \\
\hline Position 24 & 100 & 100 \\
\hline Position 25 & 100 & 100 \\
\hline Position 26 & 0 & 100 \\
\hline Position 27 & 100 & 100 \\
\hline Position 28 & 100 & 100 \\
\hline Position 29 & 100 & 100 \\
\hline
\end{tabular}
tion module is achieved through LabVIEW, and the signal processing is carried out using MATLAB.

The described materials have been employed to perform measurements on a test bench situated in the hall of laboratory work at the National School of Engineers of Le

Table 2 Precision distribution for $8.5-\mathrm{cm}$ accuracy related to the first configuration with $M \in\{7,10\}$ 


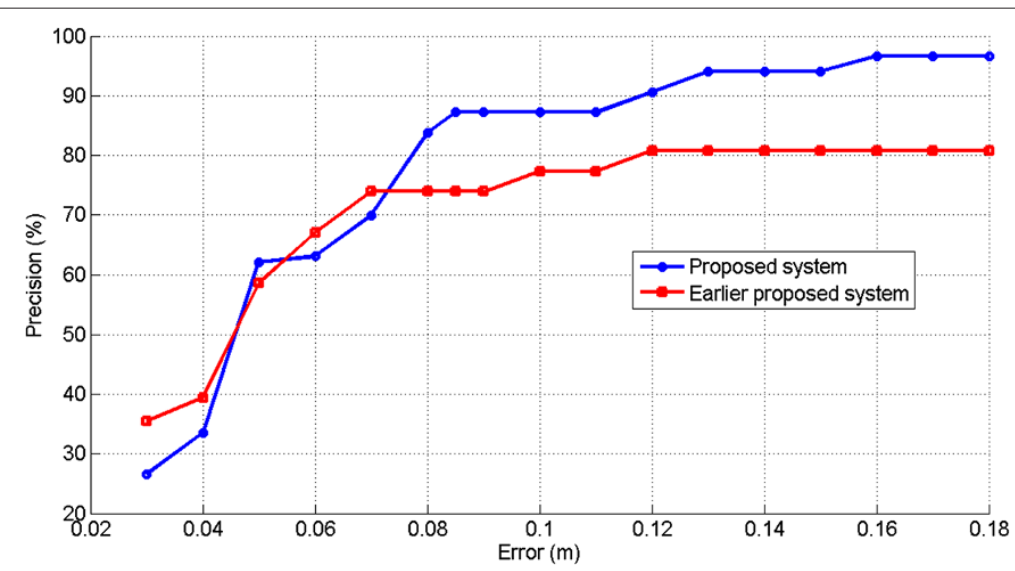

Figure 11 Cumulative error distribution functions related to the first configuration with $(M=7$ and $M=10)$.

Mans. As shown in Figure 5, the test bench consists of computer areas, desks, and different materials. Moreover, it includes controlled mechanical ventilation that generates a cyclic noise of about $25 \mathrm{~Hz}$ (Figure 1). The effective area, where we perform the experiment, measures $1.5 \mathrm{~m} \times$ $1.5 \mathrm{~m} \times 0.75 \mathrm{~m}$.

Recall that the test bench described in our earlier proposed localization scheme [15] was encircled with a curtain. The ceiling and floor were covered with a material of low reflectivity and carpet, respectively. In this paper, no arrangement was made to reduce the effects of reflections and exterior influences on the test bench.

Our system variables were set to:

- a sampling frequency of $51,200 \mathrm{~Hz}$,

- a carrier and chip frequency of $3,000 \mathrm{~Hz}$,

- a length of the gold code sequence of 127 chips and

- grid training (i.e., distance between two fingerprint positions) of $30 \mathrm{~cm}$.

System performance was evaluated in two configurations: privacy-oriented configuration and centralized configuration. Performance is given in terms of precisionversus-accuracy plots and in terms of precision distributions in the work area. The accuracy is defined as the highest tolerated error for a measure to be regarded as successful. The precision is the ratio of measurements with errors equal to or less than the accuracy.

In the next section, we present the performance of the privacy-oriented system.

Table 3 Positioning accuracy and precision related to the first configuration with four speakers

\begin{tabular}{lccccc}
\hline \multicolumn{5}{c}{ Experimental results } \\
\hline Error distance $(\mathrm{cm})$ & 5 & 6 & 8.5 & 12 & 15 \\
Precision (\%) & 27.58 & 32.01 & 37.93 & 48.27 & 58.12 \\
\hline
\end{tabular}

\section{Privacy-oriented system}

In the privacy-oriented configuration, three tweeters having known locations emit simultaneously BPSKmodulated gold codes. The microphone receives the aggregate signal, and the signal is correlated with the sources' codes to determine its signature. The position is then estimated through the local linear estimator based on the built database.

The system performance is evaluated for the setups shown in Figures 6 and 7.

The difference between the two setups is the number of deployed tweeters and positions. In this configuration, 29 positions randomly distributed in the effective area and at a height of $0.28 \mathrm{~m}$ are picked and more than 30 measurements were made at each position. However, we note that we obtain the same results if we only consider seven measurements. In this paper, we present the results obtained with seven measurements.

\subsection{Experimental results obtained with the first setup} In this subsection, we present the performance of the privacy-oriented system evaluated with the setup shown in Figure 6.

The percentage of realizations as a function of the location estimation accuracy is reported in Table 1 . The proposed system provides accuracy of about $8.5 \mathrm{~cm}$ almost $80 \%$ of the time.

Table 4 Positioning accuracy and precision related to the first configuration

\begin{tabular}{lccccc}
\hline & \multicolumn{5}{c}{ Experimental results } \\
\hline Error distance (cm) & 5 & 6 & 8.5 & 12 & 15 \\
$\begin{array}{l}\text { Precision (\%) obtained } \\
\text { with four speakers }\end{array}$ & 27.58 & 32.01 & 37.93 & 48.27 & 58.12 \\
$\begin{array}{l}\text { Precision (\%) obtained } \\
\text { with three best speakers }\end{array}$ & 35.96 & 51.72 & 61.57 & 68.96 & 74.38 \\
\hline
\end{tabular}




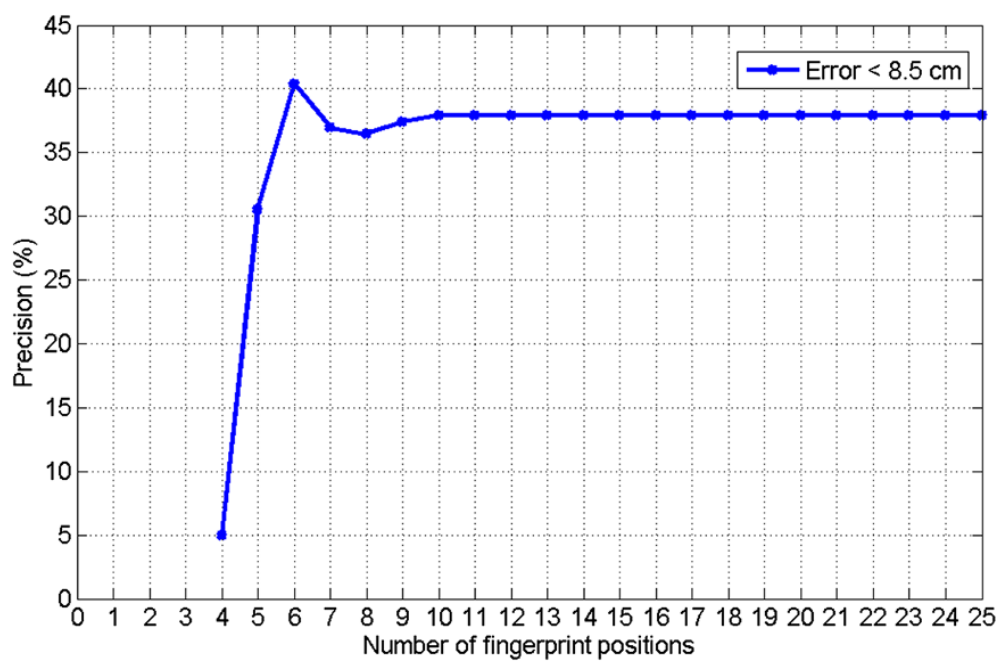

Figure 12 Precision for $8.5-\mathrm{cm}$ accuracy related to the first configuration using four speakers.

In the following, we study the effect of the number of fingerprint positions on system performance.

\subsubsection{Influence of the number of fingerprint positions on system performance}

Here, only the $M$ best fingerprint positions, according to the kernel weight $K_{H}\left(s_{i}-s\right)$, contribute to position estimation. We recall that $s_{i}$ denotes the signature of the fingerprint location $i$, and $s$ denotes the fingerprint of the microphone to be localized. As seen in Figure 8, for $M \geq$ 7 , the precision for $8.5 \mathrm{~cm}$ accuracy is $80 \%$. This means that the remaining fingerprint positions do not contribute to the position estimation. As explained earlier, this is due to the Gaussian kernel function that assigns a small weight when the value $\left(s_{i}-s\right)$ is high.
We then examine the effect of the $M$ best fingerprint positions on the precision distributions in the work area. Percentages of measurements per position for an accuracy of $8.5 \mathrm{~cm}$ obtained with the seven and ten best fingerprint positions are respectively shown in Figures 9 and 10. The numerical results extracted from these figures are reported in Table 2.

It is observed that 23 positions have a precision of $100 \%$ in both cases. However, it is important to note that the same results are obtained for 21 positions and only two positions have different results. We observe that the value of $M=10$ fingerprint positions is more suited to estimating positions at the center of the work space and the value of $M=7$ fingerprint positions is preferred in computing positions at the edges of the effective area.

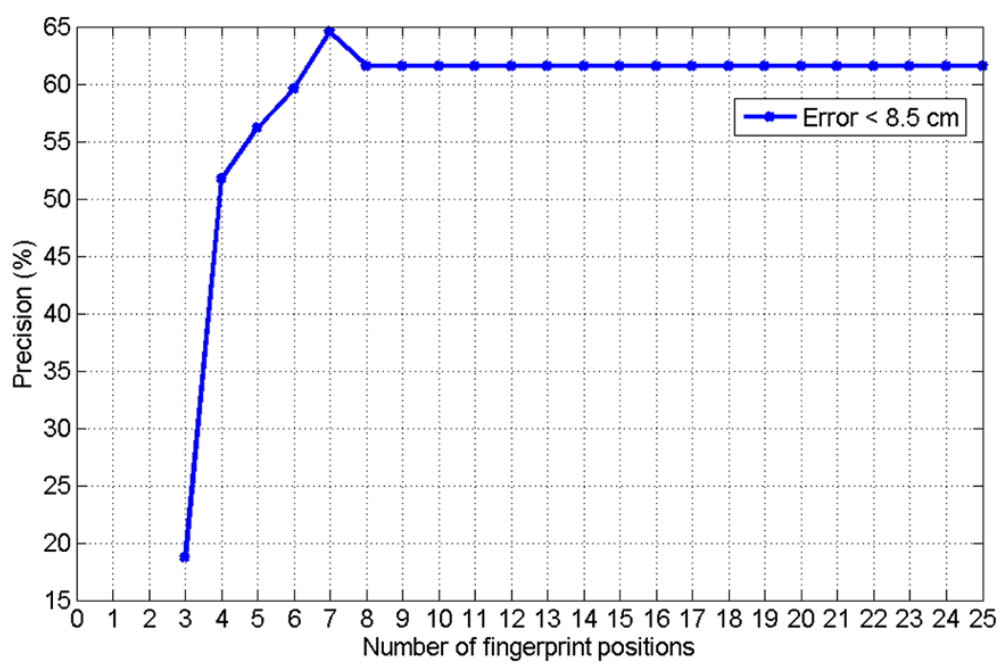

Figure 13 Precision for $8.5-\mathrm{cm}$ accuracy related to the first configuration using three best speakers. 
Table 5 The cumulative error distributions related to the first configuration

\begin{tabular}{lccccc}
\hline & \multicolumn{5}{c}{ Experimental results } \\
\hline Error distance (cm) & 5 & 6 & 8.5 & 12 & 15 \\
$\begin{array}{l}\text { Precision (\%) with four } \\
\text { speakers and M=6 }\end{array}$ & 26.60 & 32.51 & 40.39 & 44.33 & 48.76 \\
$\begin{array}{l}\text { Precision (\%) with three } \\
\text { best speakers and M=7 }\end{array}$ & 43.84 & 53.69 & 64.53 & 70.44 & 72.90 \\
\hline
\end{tabular}

Therefore, we obtain 25 positions with a precision of $100 \%$ and $87.19 \%$ of total measurements having an error below $8.5 \mathrm{~cm}$.

Next, a comparison between the obtained performance and that of our earlier proposed system [15] is conducted.

\subsubsection{Cumulative error distribution}

The system presented in [15] deploys the TOA of CDMA signals and employs lateration to locate a microphone. The TOA is estimated by correlating the received signal with the tweeter codes. To compare objectively the system in [15] with the proposed system, we evaluated its performance on our test bench. The cumulative error distribution functions of both systems are depicted in Figure 11.

As shown in Figure 11, the proposed system has localization accuracy below $8.5 \mathrm{~cm}$ for $87 \%$ of measurements, while the earlier proposed system achieves an error of $12 \mathrm{~cm}$ with $80 \%$ precision. However, it should be noted that for error within $7 \mathrm{~cm}$, the cumulative error distribution functions of both systems are close, and that beyond this value, the described system outperforms the system presented in [15].

On the other hand, it is worth noting that our earlier proposed system [15] has less than expected performance when considering the results reported in [15]. This is because arrangements have been taken to reduce the effects of reflections and exterior influences on the test bench presented in [15]. The test bench described here is a part of the real world and has not been modified to evaluate the system performance.

In the following, we will evaluate the system performance under a varying number of tweeters and their positions.

\subsection{Experimental results obtained with the second setup}

The privacy-oriented system was evaluated with four speakers as shown in Figure 7. We kept the same parameters as in the first setup (Figure 6). However, it should be noted that some measurements are made when some trainees are around the effective area.

The obtained results are reported in Table 3 . They show degradation in precision of about $40 \%$ relative to the results obtained in the case of three speakers. This poorer performance can be explained by the increase in the nearfar effect and multiple access interference due to the addition of a supplementary speaker. On the other hand, the speaker placements may contribute to this degradation. In fact, compared with the first setup, we have two additional speakers that are near the table and wall. This arrangement may generate paths of similar amplitudes and thus degrade the estimation of the TOA of the path of maximum amplitude.

To improve the obtained results, we attribute to each speaker a peak quality parameter. Only the three best speakers, according to this criterion, will contribute to position estimation. This means that only the three selected speakers in the target fingerprint that are also contained in the database are employed to compute the microphone position. The peak quality parameter is defined as:

$$
q_{i}=\max \left(R_{r, s_{i}}\right)
$$

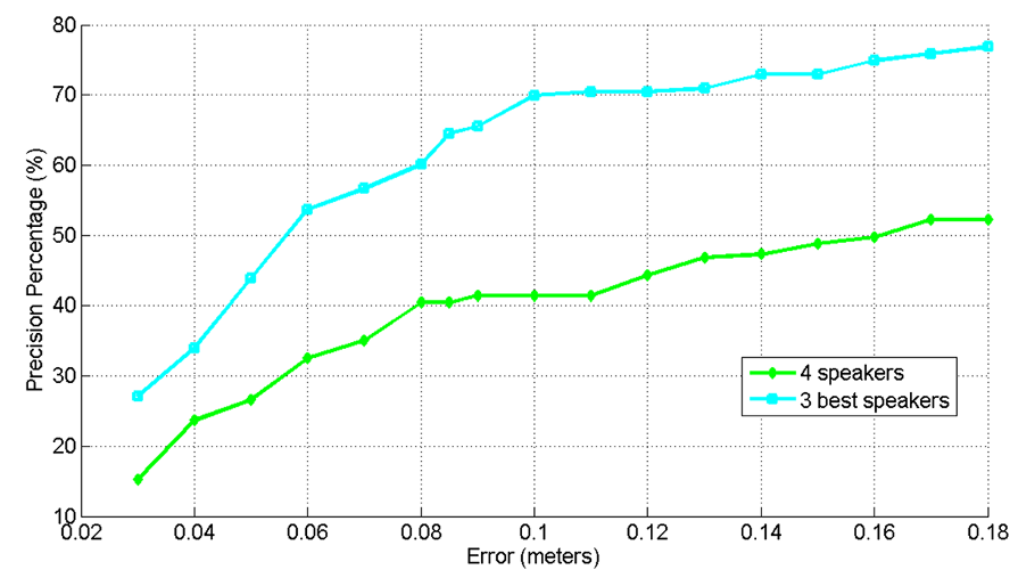

Figure 14 Precision versus location estimation accuracy related to the first configuration. 


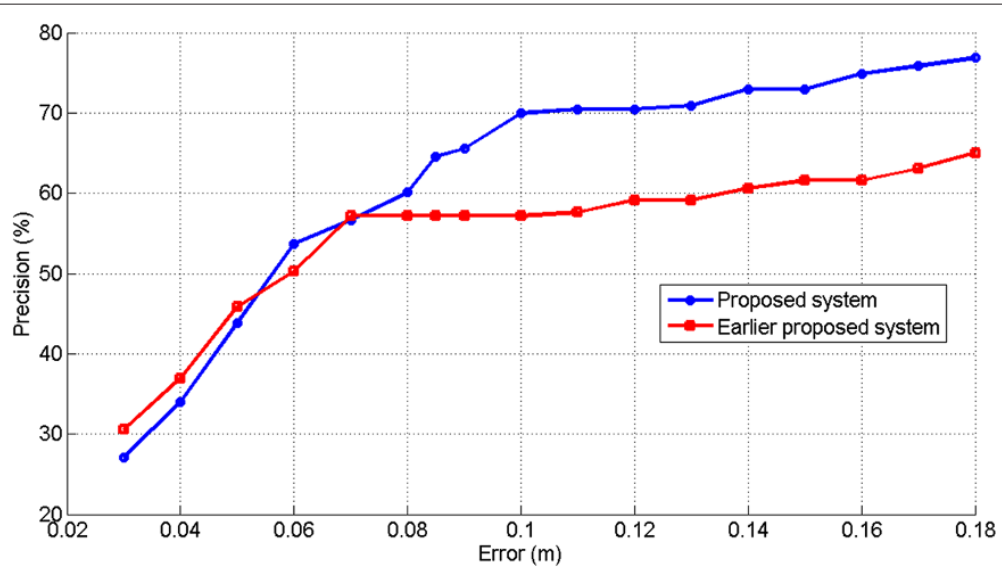

Figure 15 Cumulative error distribution functions related to the first configuration using three best speakers and $M=7$.

where $R_{r, s_{i}}$ is the cross-correlation between the received signal $r$ and the signal $\left(s_{i}\right)$ from the $i$ th speaker. Note that this criterion was proposed in [15], but it was not used.

Results, reported in Table 4, show enhancement in precision of about $24 \%$ when comparing the case of the three best speakers with that of the four speakers.

As in the first setup, the effect of the number of fingerprint positions on system performance will be studied when employing four speakers.

\subsubsection{Influence of the number of fingerprint positions on system performance}

Here, we investigate the effect of the number of fingerprint positions on system performance when using the three best speakers and when using four speakers. As shown in Figures 12 and 13, the best precision is obtained with $M=6$ and $M=7$, respectively, for four speakers and the three best speakers.
For further insight into the evaluation results, the cumulative error distributions obtained with the best number of fingerprint positions are illustrated in Table 5 and plotted in Figure 14.

The system provides an error below $8.5 \mathrm{~cm}$ for $64.5 \%$ and $40 \%$ of measurements, respectively, with the three best speakers and four speakers. Although the addition of a supplementary source provided additional information for location estimation, it worsened the system performance. In fact, the estimation of the TOA of the path of maximum amplitude was degraded by interference and by emitter placement. Hence, we find a trade-off between redundancy and interference, relative to our setup.

Moreover, the selection of the best number of fingerprint positions improved the precision by $3 \%$.

In the following, we compare the performance of the described system with that of our earlier proposed scheme [15].

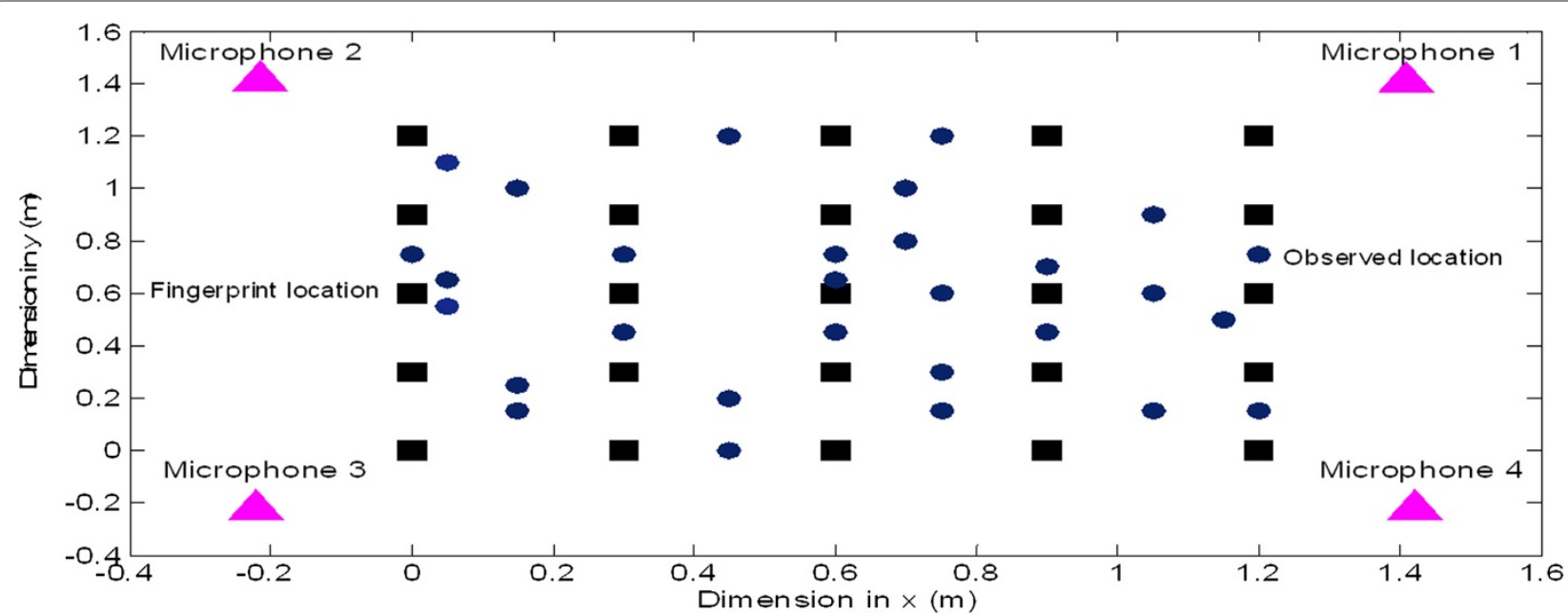

Figure 16 Layout of the experiment setup in the case of the second configuration. 
Table 6 Precision versus location estimation accuracy obtained in the second configuration

\begin{tabular}{lcccccccccccc}
\hline \multicolumn{10}{c}{ Experimental results } \\
\hline Error distance (cm) & 1 & 1.3 & 1.4 & 1.6 & 1.8 & 1.9 & 2 & 2.1 & 2.2 & 2.6 & 2.7 & 3 \\
Precision (\%) & 57.3 & 62.6 & 62.6 & 69.3 & 74.6 & 78.6 & 78.6 & 82.6 & 86.6 & 89.3 & 93.3 & 93.3 \\
\hline
\end{tabular}

\subsubsection{Cumulative error distribution}

A comparison between the cumulative error distribution function of the described system and that of our earlier proposed system [15], evaluated on the same test bench and obtained with the three best speakers, is presented in Figure 15. The proposed system has localization accuracy below $8.5 \mathrm{~cm}$ for $64 \%$ of measurements, whereas the earlier proposed system has error below $18 \mathrm{~cm}$ for the same percentage of measurements. As in the case of the first setup, the described system outperforms the earlier proposed system for errors greater than $7 \mathrm{~cm}$.

\subsection{Summary of the privacy-oriented system}

Results obtained in both setups show that our location system is more suitable to areas with a limited number of speakers. This is expected for CDMA systems. However, by selecting the three best speakers, the system still presents acceptable results. On the other hand, our system performs better than that presented in [15] on the same test bench.

In the next section, the system will be evaluated in a centralized configuration using TDMA operation.

\section{Centralized system}

In the centralized configuration, four microphones at known positions are deployed to locate the tweeter position (see Figure 16). The received signals are correlated with the emitted gold code to obtain the source signature. The position is estimated through the local linear estimator.

Notice that, in this configuration, we have deployed four microphones instead of three to provide additional location information while no interference is present.

Results are given in the next section.

\subsection{Experimental results}

The percentage of realizations as a function of the location estimation accuracy is listed in Table 6. The proposed system has accuracy of about $2.7 \mathrm{~cm}$ almost $93.3 \%$ of the time.

Compared with the performance of the privacyoriented system, the centralized system has better accuracy. This is because neither multiple access interference nor the near-far effect is present.

In the following, we study the effect of the number of fingerprint positions on system performance.

\subsubsection{Influence of the number of fingerprint positions on system performance}

The effect of the number of fingerprint positions is investigated. As shown in Figure 17, for $M \geq 6$, the precision for $2.7 \mathrm{~cm}$ accuracy is $93.3 \%$.

Precision versus accuracy for $M=7$ is illustrated in Table 7. An increase in precision of almost 3\% is achieved relative to results obtained with all fingerprint positions.

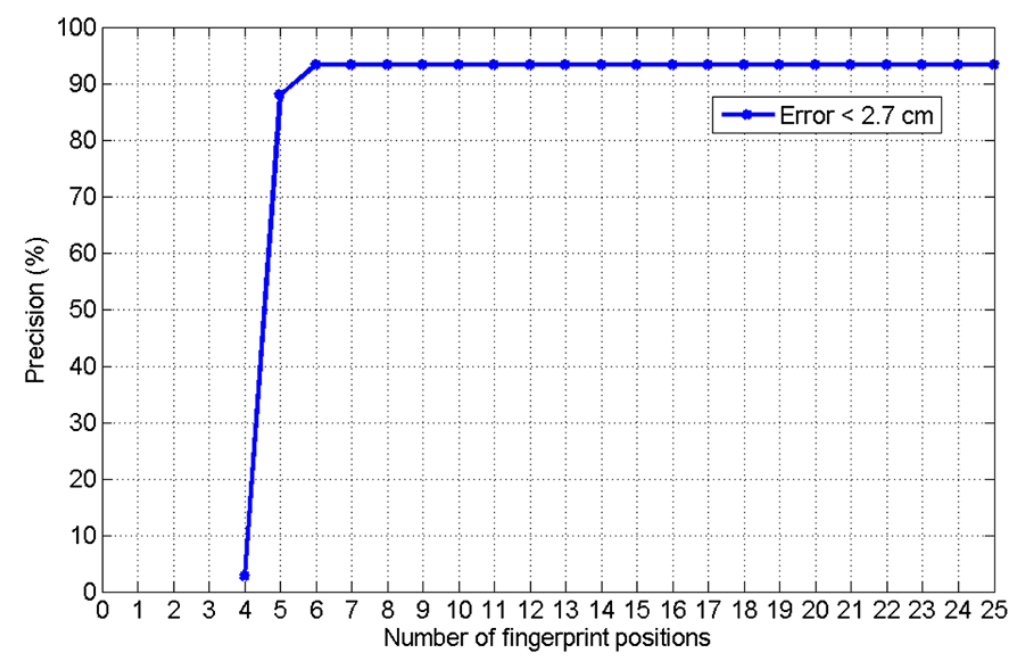

Figure 17 Precision for $2.7-\mathrm{cm}$ accuracy versus number of fingerprint positions related to the second configuration. 
Table 7 Precision versus accuracy obtained with $M=7$ in the case of the second configuration

\begin{tabular}{lccccccccccccc}
\hline \multicolumn{10}{c}{ Experimental results } \\
\hline Error distance (cm) & 1 & 1.3 & 1.4 & 1.6 & 1.8 & 1.9 & 2 & 2.1 & 2.2 & 2.6 & 2.7 & 3 \\
Precision (\%) & 57.3 & 62.6 & 66.6 & 70.6 & 74.6 & 78.6 & 81.3 & 85.3 & 86.6 & 89.3 & 93.3 & 93.3 \\
\hline
\end{tabular}

\subsubsection{Precision distributions}

Precision per position is depicted in Figure 18. All but two test positions have precision of $100 \%$ for $2.7 \mathrm{~cm}$ accuracy; one position has precision of $0 \%$ and a second has precision of $33.3 \%$.

\subsection{Summary of the centralized system}

The centralized system provides better accuracy than the privacy-oriented system. However, unlike the centralized system, the privacy-oriented system has the ability to localize many receivers at the same time.

\section{Discussion and future work}

We discuss limitations of our work and present our future work.

It is first worth noting that the dimensions of our test bench are limited by the materials available at the National School of Engineers of Le Mans. One way of using our system in the real world is to fix the sources of the privacyoriented system and the receivers of the centralized system on the ceiling. The height of fingerprint locations should be close to that of the target.

Moreover, to obtain a good resolution in time for localization purposes, we need a wideband signal for exploring or propagating in the medium under test. In the case of ultrasonic signals, the transducers suffer from a narrow bandwidth with few hundreds of Hertz. In this paper, we ought to use an audible signal, while using a normal condenser microphones and magnet-based loudspeakers with bandwidths as wide as $10 \mathrm{KHz}$. However, these signals became audible. For such problem, our solution is to spread the bandwidth of the signal by scrambling using a
CDMA code, which will reduce the power spectral density as the energy of the signal is spreaded over the useful bandwidth. Hence, these coded signals become almost nonaudible.

On the other hand, we have assumed that the emitters are synchronized with the receivers. RF signals can be employed to achieve the synchronization [10].

In future work, we aim to estimate the position of the target in three dimensions. Hence, we should place the fingerprint positions at different values of $x, y$, and $z$, which will yield additional training data. To reduce the number of fingerprint positions, we will consider a nonregular grid; i.e., the fingerprint positions are not regularly spaced. On the other hand, the number of emitters of the privacyoriented system will be selected for good performance. The authors of [19] found that using more fixed nodes in the $3 \mathrm{D}$ scenario than in the $2 \mathrm{D}$ scenario provided minimal improvement.

We will conduct experiments for the $3 \mathrm{D}$ scenario and report the relationship between localization accuracy and fingerprint size (i.e., the number of emitters deployed in the privacy-oriented system) to determine the number of emitters that is the most appropriate for our system [14].

Moreover, our work in this paper focused on the experimental evaluation of system performance. Theoretical limits for the accuracy of location estimates will be the object of further investigation.

\section{Conclusion}

This paper presented an acoustic localization system based on the TOA and fingerprinting technique. The system was evaluated in two configurations: a privacy-

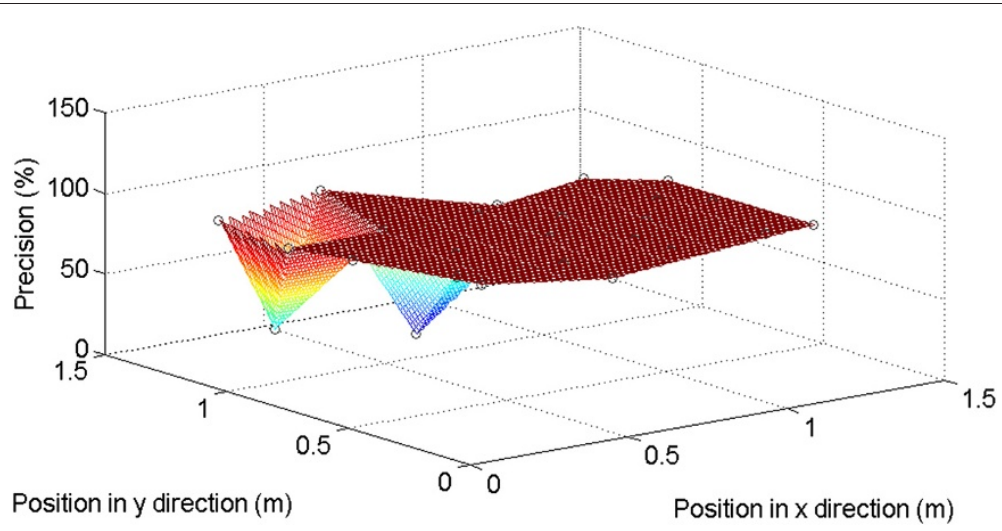

Figure 18 Precision distribution for $2.7-\mathrm{cm}$ accuracy related to the second configuration using $M=7$. 
oriented system with CDMA operation and a centralized system with TDMA operation. The effects of the number and positions of emitters were studied for the privacyoriented system, and the effect of the number of fingerprint positions on system performance was investigated for both configurations. A deterioration of performance of the privacy-oriented system was observed when adding a supplementary source and changing the emitter placement. However, this was alleviated by selecting the three best sources for the position estimation. On the other hand, the choice of the best number of fingerprint positions improved the precision by $3 \%$ and $7 \%$ for centralized and privacy-oriented systems, respectively.

To objectively compare the privacy-oriented system and lateration-based system presented in [15], we evaluated the system in [15] on our test bench. The cumulative error distribution function of our system is revealed to be better. This, we believe, is an interesting contribution to the literature, as it demonstrates the effect of reverberation on naive acoustic localization.

\section{Competing interests}

The authors declare that they have no competing interests.
12. JC Prieto, AR Jiménez, J Guevara, JL Ealo, F Seco, JO Roa, F Ramos, Performance evaluation of 3D-LOCUS advanced acoustic LPS. IEEE Trans. Instrum. Meas. 58, 2385-2395 (2009)

13. M Azizyan, I Constandache, RR Choudhury, SurroundSense: Mobile Phone Localization via Ambience Fingerprinting. (ACM, New York, 2009)

14. SP Tarzia, PA Dinda, RP Dick, G Memik, Indoor Localization without Infrastructure using the Acoustic Background Spectrum, in Proceedings of the 9th International Conference on Mobile Systems Applications and Services: 28 Jun-1 Jul 2011; Bethesda (ACM, New York, 2011)

15. C Sertatila, MA Altinkayaa, K Raoof, A novel acoustic indoor localization system employing CDMA. Digital Signal Process. 22, 506-517 (2002)

16. N Aloui, K Raoof, A Bouallegue, S Letourneur, S Zaibi, A novel indoor localization scheme based on fingerprinting technique And CDMA signals, in 2012 International Conference on Indoor Positioning and Indoor Navigation: 13-15 Nov 2012; Sydney (IEEE, Piscataway, 2012), pp. 1-5

17. W Hardle, M Muller, S Sperlich, A Werwatz, Nonparametric and Semiparametric Models, in Springer Series in Statistics (Springer, Heidelberg, 2004)

18. MG Schimek, Smoothing and Regression Approaches, Computation, and Application. (Wiley, Hoboken, 2000)

19. M Giuberti, M Martalò, G Ferrari, Fingerprinting-based wireless 3D localization for motion capture applications, in MobileHealth '11 Proceedings of the First ACM MobiHoc Workshop on Pervasive Wireless Healthcare (ACM, New York, 2011)

doi:10.1186/1687-6180-2014-13

Cite this article as: Aloui et al:: Performance evaluation of an acoustic indoor localization system based on a fingerprinting technique. EURASIP Journal on Advances in Signal Processing 2014 2014:13.

\begin{abstract}
Author details
${ }^{1}$ Grenoble Images Parole Signal Automatique (GIPSA-lab) CNRS : UMR5216 Université Joseph Fourier - Grenoble I - Université Pierre-Mendès-France Grenoble II - Université Stendhal - Grenoble III - Institut Polytechnique de Grenoble (Grenoble Institute of Technology), Grenoble, France. ${ }^{2}$ Université de Tunis El Manar, Ecole Nationale d'Ingénieurs de Tunis, LR-99-ES21 Laboratoire de Systèmes de Communications, Tunis 1002, Tunisie. ${ }^{3}$ LAUM Laboratory UMR CNRS 6613, University of Maine, Le Mans 72085, France.
\end{abstract}

Received: 4 May 2013 Accepted: 15 January 2014

Published: 30 January 2014

\section{References}

1. Y Gu, A Lo, I Niemegeers, A survey of indoor positioning systems for wireless personal networks. IEEE Commun. Surv. Tutorials. 11, 13-31 (2009)

2. M Hazas, A Hopper, Broadband ultrasonic location systems for improved indoor positioning. IEEE Trans. Mobile Comput. 5, 536-547 (2006)

3. X Fernando, S Krishnan, H Sun, K Kazemi-Moud, Adaptive Denoising at Infrared Wireless Receivers (SPIE, Bellingham, 2003)

4. I Rishabh, D Kimber, J Adcock, Indoor Positioning System Based on Incident Angles of Infrared Emitters (Industrial Electronics Society, Busan, 2004)

5. R Want, A Hopper, V Falcao, J Gibbons, The active badge location system ACM Trans. Inf. Syst. 10, 91-102 (1992)

6. S Dayekh, S Affes, N Kandil, C Nerguizian, Cooperative localization in mines using fingerprinting and neural networks, in 2010 IEEE Wireless Communications and Networking Conference (WCNC): 18-21 Apr 2010; Sydney (IEEE, Piscataway, 2010), pp. 1-6

7. Y Jin, W-S Soh, Member, W-C Wong, Indoor localization with channe impulse response based fingerprint and nonparametric regression. IEEE Trans. Wireless Commun. 9, 1120-1127 (2010)

8. A Kushki, KN Plataniotis, AN Venetsanopoulos, Kernel-based positioning in wireless local area networks. IEEE Trans. Mobile Comput. 6, 689-705 (2007)

9. C Nerguizian, C Despins, S Affes, Geolocation in mines with an impulse response fingerprinting technique and neural networks. IEEE Trans. Wireless Commun. 5, 603-611 (2006)

10. NB Priyantha, A Chakraborty, H Balakrishnan, The Cricket location-support system, in Proc. ACM conference On Mobile Computing and Networking (ACM, New York, 2000)

11. NB Priyantha, The Cricket Indoor Location System. PhD thesis, MIT, 2005

\section{Submit your manuscript to a SpringerOpen ${ }^{\mathcal{O}}$ journal and benefit from:}

- Convenient online submission

- Rigorous peer review

- Immediate publication on acceptance

- Open access: articles freely available online

- High visibility within the field

- Retaining the copyright to your article

Submit your next manuscript at $>$ springeropen.com 